\title{
Qualidade de vida de mulheres com dor pélvica crônica*
}

\section{Quality of life of women with chronic pelvic pain}

Rosa Azevedo da Luz ${ }^{1}$, Flávia Melo Rodrigues ${ }^{2}$, Vanessa Silva Carvalho Vila ${ }^{3}$, José Miguel de Deus ${ }^{4}$, Délio Marques Conde ${ }^{5}$

\footnotetext{
* Trabalho realizado no Ambulatório de Ginecologia do Hospital das Clínicas da Universidade Federal de Goiás.

${ }^{1}$ Enfermeira, Mestre em Ciências Ambientais e Saúde. Enfermeira do Hospital das Clínicas da Universidade Federal de Goiás (UFG). Goiânia, GO, Brasil. E-mail: r.azevedo.luz@bol.com.br.

${ }^{2}$ Biomédica, Doutora em Ciências Ambientais. Professora Adjunto da Pontifícia Universidade Católica de Goiás (PUC-GO). Goiânia, GO, Brasil. E-mail: rflamelo@gmail.com.

${ }^{3}$ Enfermeira, Doutora em Enfermagem. Professora Adjunto da PUC-GO. Goiânia, GO, Brasil. E-mail: vscvila@uol.com.br.

${ }^{4}$ Médico, Doutor em Medicina. Professor Associado da Faculdade de Medicina da UFG. Goiânia, GO, Brasil. E-mail: jm.dedeus@hotmail.com.

${ }^{5}$ Médico, Doutor em Tocoginecologia. Preceptor do Programa de Residência Médica do Hospital Materno Infantil de Goiânia. Goiânia, GO, Brasil. E-mail: delioconde@bol.com.br.
}

\section{RESUMO}

Os objetivos deste estudo transversal foram comparar a qualidade de vida e as características socioeconômicas de mulheres com e sem dor pélvica e investigar achados clínicos e cirúrgicos de mulheres com dor pélvica crônica. Os dados foram coletados em um hospital público, de Goiânia/GO, Brasil. A intensidade da dor foi aferida pela escala analógica visual e qualidade de vida avaliada pelo SF-36. A maioria das mulheres classificou a dor como intensa (52\%) e conviviam com a dor há $8,8( \pm 7,7)$ anos. Mulheres do grupo com dor pélvica crônica apresentaram escores inferiores em todas as dimensões avaliadas pelo SF-36 ( $p<0,05)$, exceto na dimensão saúde mental, quando comparado com o grupo sem dor. A correlação foi negativa $(p=0,017)$ entre a intensidade da dor e a dimensão dor do SF-36. A dor pélvica apresentou impacto negativo significativo na qualidade de vida, com prejuízos para a saúde física e mental das mulheres.

Descritores: Qualidade de Vida; Dor Pélvica; Saúde da Mulher; Cuidados de Enfermagem.

\section{ABSTRACT}

The objective of this cross-sectional study was to compare the quality of life and socioeconomic characteristics of women with and without pelvic pain and investigate clinical and surgical findings about women with chronic pelvic pain. Data were collected in a public hospital in Goiânia, Goiás, Brazil. Pain intensity was measured using the visual analogical scale, whereas quality of life was assessed through the SF-36. Most women classified pain as intensive (52\%) and lived with pain for $8.8( \pm 7.7)$ years. Women belonging to the group of chronic pelvic pain scored less in all dimensions evaluated by the SF-36 ( $p<0.05)$, except for the mental health dimension, when compared to the painless group. Correlation was negative $(p=0,017)$ between pain intensity and the SF-36 bodily pain dimension. Pelvic pain presented significant negative impact on quality of life with losses to the physical and mental health of women.

Descriptors: Quality of Life; Pelvic Pain; Women's Health; Nursing Care. 


\section{INTRODUÇÃO}

Entre as condições crônicas de saúde vivenciadas por mulheres em idade reprodutiva destaca-se a dor pélvica crônica (DPC), definida como dor acíclica, constante ou intermitente, localizada no abdômen inferior ou pelve, com duração de no mínimo seis meses, não associada exclusivamente à menstruação, à relação sexual ou à gravidez $^{(1-3)}$.

As evidências científicas apontam a DPC como um problema de saúde pública que gera custos diretos e indiretos para a saúde da mulher e para os sistemas de saúde ${ }^{(4)}$. Nos Estados Unidos, os gastos anuais estimados para o tratamento de mulheres com DPC foi em torno de $\$ 881,5$ milhões de dólares ${ }^{(4)}$ e na Inglaterra esse gasto anual foi estimado em $£ 158$ milhões de libras ${ }^{(4)}$.

A DPC é uma das condições crônicas mais comuns vivenciadas pelas mulheres que poderá afetar gravemente sua qualidade de vida (QV), incluindo aspectos relacionados ao funcionamento físico, o bemestar psicológico e as relações interpessoais ${ }^{(5)}$. Outros estudos descreveram a DPC como condição crônica debilitante, causadora de sofrimento, que pode levar a alterações emocionais tais como, depressão, ansiedade, insônia e disfunções sexuais, além de afetar a QV das mulheres $^{(3,6-8)}$.

Contudo, ainda são escassos os estudos de base populacional sobre a prevalência da DPC no mundo e seus efeitos sobre a qualidade de vida e a saúde das mulheres. A prevalência da DPC é difícil de ser determinada, pois está associada à grande variação de sua definição clínica ${ }^{(5)}$. Uma revisão da Organização Mundial de Saúde (OMS) realizada pelo Latthe e colaboradores mostrou que a prevalência a nível mundial desta condição varia de $2 \%$ a $24 \%{ }^{(9)}$.

Estudos prévios descreveram a estimativa da prevalência da DPC nas mulheres entre $12 \%$ a $39 \%{ }^{(5,10)}$. Entretanto, um estudo de revisão sistemática recente encontrou taxas de prevalência da DPC entre 5,7\% a $26,6 \%$, incluindo países que anteriormente nunca tiveram publicações de estudos de base populacional sobre prevalência da DPC como: Gana, Egito, Áustria, Austrália e Brasil ${ }^{(11)}$. Esse estudo afirma que há muitos países e regiões sem dados básicos sobre a DPC e refere essa ausência, talvez, ao fato dos profissionais de saúde e pesquisadores não terem interesse em realizar estudos, devido à DPC ser de natureza complexa e à falta de conhecimento a respeito desta temática.

Embora a DPC seja um problema mundial que afeta mulheres numa ampla faixa etária, os dados disponíveis, sobre sua prevalência, ainda são restritos e, no Brasil, foi identificado um único estudo até o momento que investigou mulheres com idade acima de 14 anos e mulheres em idade reprodutiva e encontrou taxas de $11,5 \%$ e $15,1 \%$, respectivamente ${ }^{(12)}$.

A DPC tem etiologia pouco esclarecida ${ }^{(6)}$ e pode estar relacionada a processos crônicos de uma complexa interação entre os sistemas gastrintestinal, urológico, genital e músculo-esquelético ${ }^{(6,13)}$. Além do mais, as mulheres com DPC comumente apresentam doenças coexistentes, tais como endometriose, doença inflamatória pélvica, aderências pélvicas, síndrome do cólon irritável, cistite intersticial e fibromialgia ${ }^{(6,10,13-14)}$.

Considerando que a DPC é uma importante causa de morbidade em mulheres ${ }^{(9)}$ que poderá trazer limitações para a funcionalidade e bem-estar e, ainda, são raros, especialmente no Brasil, os estudos que avaliam a qualidade de vida relacionada à saúde de mulheres com DPC ${ }^{(8,15-16)}$. O presente estudo foi realizado com o objetivo de comparar a QV e as características sociodemográficas e econômicas de mulheres com e sem dor pélvica crônica e investigar os achados clínicos e cirúrgicos de mulheres com dor pélvica crônica.

\section{MATERIAL E MÉTODO}

Este estudo de corte transversal analítico foi realizado no ambulatório de ginecologia, de uma instituição hospitalar de grande porte, referência no atendimento em DPC pelo Sistema Único de Saúde (SUS), no município de Goiânia-GO, Brasil. Os dados foram coletados no período de outubro de 2009 a maio de 2010. 
A amostra desse estudo foi constituída por 100 mulheres, em dois grupos denominados com e sem dor pélvica crônica. O cálculo amostral foi baseado na amostragem representativa ${ }^{(17)}$ que é dado por: $n_{0}=1 / E_{0}{ }^{2}$; $\mathrm{n}=\mathrm{N} \times \mathrm{n}_{0} / \mathrm{N}+\mathrm{n}_{0}$, em que $\mathrm{n}_{0}$ é a primeira aproximação do tamanho amostral, E é o erro amostral tolerável $(0,1)$, n é o tamanho da amostra e N é o tamanho da população (96 pacientes/mês no período da coleta de dados). Portanto, 50 foi o tamanho da amostra representativa para cada grupo.

Os critérios de inclusão das mulheres no grupo com DPC foram: ter idade igual ou superior a 18 anos e diagnóstico de dor pélvica crônica. No grupo sem DPC, os critérios foram: ter idade igual ou superior a 18 anos; estar em seguimento no ambulatório de planejamento familiar e não ter queixa de DPC. Nos dois grupos, foram excluídas as mulheres com antecedentes de gravidez, nos últimos 12 meses ou em tratamento de neoplasia maligna.

A pesquisa foi aprovada pelo Comitê de Ética em Pesquisa da instituição na qual o estudo foi conduzido, sob o protocolo no 082/2009 e obedeceu aos aspectos éticos legais da legislação brasileira para pesquisa com seres humanos. Todas as participantes aceitaram participar e assinaram o Termo de Consentimento Livre e Esclarecido (TCLE).

A coleta de dados foi realizada por meio de entrevista estruturada, na qual foram coletados dados sociodemográficos, econômicos, características clínicas da dor (local da dor, fator de piora e melhora, tempo de dor, se a dor está relacionada ao período menstrual, se apresenta disúria e pratica atividade física), dados relacionados ao tratamento cirúrgico da dor (número de cirurgias realizadas relacionadas à DPC, se a vida melhorou com o tratamento cirúrgico e outras cirurgias realizadas).

A intensidade da dor foi investigada por meio da Escala Analógica Visual (EAV) a qual é unidimensional, bastante usada na prática clínica e de fácil compreensão ${ }^{(18)}$. Essa escala é graduada de zero a 10; o zero corresponde à ausência de dor e 10, a pior dor possível. Essa escala avalia a intensidade da dor, classificando-a em ausência de dor (zero), dor leve (um a três), dor moderada (quatro a sete) e dor intensa (oito a dez) ${ }^{(18)}$.

Para avaliação da QV, aplicou-se o questionário Medical Outcomes Study $36^{\circledR}$ - Item Short Form Health Survey(SF-36) ${ }^{(19)}$. Trata-se de um instrumento genérico, multidimensional, de avaliação da QV e possui propriedades psicométricas confiáveis ${ }^{(19)}$. Foi traduzido e validado para a língua portuguesa do Brasil ${ }^{(20)}$. Esse instrumento foi utilizado em vários estudos, tendo sido aplicado a diversos tipos de doenças e/ou condições crônicas, nos últimos 20 anos $^{(19-20)}$. Um estudo de revisão sistemática da literatura realizado no Reino Unido identificou que o SF-36 foi o instrumento mais utilizado para a avaliação da QV de mulheres com DPC ${ }^{(21)}$.

O SF-36 avalia a percepção que a pessoa tem de sua vida em relação à sua saúde nas últimas quatro semanas. É composto por 36 itens, agrupados em oito dimensões: capacidade funcional, aspectos físicos, dor, estado geral de saúde, vitalidade, aspectos sociais, aspectos emocionais e saúde mental que constituem o componente da saúde física (CSF) e o componente da saúde mental (CSM). O escore do SF-36 varia de 0 a 100. O valor zero corresponde ao pior estado de saúde e 100, ao melhor estado de saúde ${ }^{(19)}$. Para cada dimensão do SF36, foram calculadas as médias dos escores e analisadas, separadamente, conforme preconizado pelos idealizadores do instrumento ${ }^{(19)}$.

Foram realizadas análises descritivas de frequência simples para as variáveis nominais de posição (média) e dispersão (desvio-padrão) para as numéricas. Utilizou-se teste $t$ de Student de comparação de médias para avaliar as diferenças significantes entre variáveis numéricas contínuas, e o coeficiente de correlação de Spearman para avaliar a associação entre as dimensões do SF-36 e a intensidade da dor. Para as demais variáveis, utilizou-se o teste do qui-quadrado. Os valores $\mathrm{p} \leq 0,05$ foram considerados estatisticamente significantes. 


\section{RESULTADOS}

A média de idade das mulheres com DPC foi de 41,65 $\pm 9,42$ anos e das mulheres sem DPC foi $28,97 \pm 6,68$ anos. As mulheres com DPC apresentavam renda mensal familiar em média de $R \$ 1.411,30 \pm 924,9$, enquanto que o grupo sem DPC foi $\mathrm{R} \$ 1.007,40 \pm 464,09$.

Em ambos os grupos, predominaram mulheres com nível médio de escolaridade, não brancas e que viviam com companheiro fixo. A maioria estava economicamente ativa, tendo como ocupação principal serviços domésticos. Essas mulheres eram responsáveis pela renda familiar e tinham dois filhos. No entanto, em relação à moradia identificou-se que a maioria das mulheres com DPC (76\%) residia em casa própria, enquanto que as mulheres sem DPC (56\%) não residiam em casa própria $(p<0,0011)$ (Tabela 1$)$.

Tabela 1: Características sociodemográficas de mulheres com e sem dor pélvica crônica. Goiânia, GO, Brasil, $2009-2010$.

\begin{tabular}{|c|c|c|c|c|c|}
\hline \multirow{2}{*}{ Características } & \multicolumn{2}{|c|}{ Com DPC $(n=50)$} & \multicolumn{2}{|c|}{ Sem DPC $(n=50)$} & \multirow{2}{*}{$p^{*}$} \\
\hline & $n$ & $\%$ & $\mathrm{n}$ & $\%$ & \\
\hline \multicolumn{6}{|l|}{ Nível de escolaridade } \\
\hline$\leq 7$ anos & 22 & 44 & 15 & 30 & \multirow[t]{2}{*}{0,1471} \\
\hline$>7$ anos & 28 & 56 & 35 & 70 & \\
\hline \multicolumn{6}{|l|}{ Raça/Etnia } \\
\hline Branca & 21 & 42 & 23 & 46 & \multirow[t]{2}{*}{0,8403} \\
\hline Não branca & 29 & 58 & 27 & 54 & \\
\hline \multicolumn{6}{|l|}{ Estado marital } \\
\hline Com companheiro & 39 & 78 & 36 & 72 & \multirow[t]{2}{*}{0,4884} \\
\hline Sem companheiro & 11 & 22 & 14 & 28 & \\
\hline \multicolumn{6}{|l|}{ Ocupação } \\
\hline Serviços domésticos & 23 & 46 & 20 & 40 & \multirow{2}{*}{0,5445} \\
\hline Outro & 27 & 54 & 30 & 60 & \\
\hline \multicolumn{6}{|l|}{ Situação de trabalho } \\
\hline Ativo & 49 & 98 & 50 & 100 & \multirow{2}{*}{0,3149} \\
\hline Inativo (aposentado e licença) & 1 & 2 & 0 & 0 & \\
\hline \multicolumn{6}{|l|}{ Responsável pela renda } \\
\hline Você & 32 & 64 & 29 & 58 & \multirow{2}{*}{0,5385} \\
\hline Outros & 18 & 36 & 21 & 42 & \\
\hline \multicolumn{6}{|l|}{ Moradia própria } \\
\hline Sim & 38 & 76 & 22 & 44 & \multirow{2}{*}{0,0011} \\
\hline Não & 12 & 24 & 28 & 56 & \\
\hline \multicolumn{6}{|l|}{ Números de filhos } \\
\hline Até dois filhos & 32 & 64 & 40 & 80 & \multirow{2}{*}{0,0748} \\
\hline Mais que dois filhos & 18 & 36 & 10 & 20 & \\
\hline
\end{tabular}

* Teste do Qui-quadrado.

Em relação aos aspectos clínicos associados à DPC, identificou-se que $52 \%$ das mulheres relataram dor intensa e, 48\%, dor moderada. A média do tempo de dor foi de $8,8 \pm 7,7$ anos. Para o alívio da dor, $78 \%$ referiram o uso de analgésicos comuns e $62 \%$ afirmaram que a vida melhorou após o uso da medicação.

Entre as mulheres com DPC, 39\% relataram dor na fossa ilíaca esquerda (FIE) e $35 \%$, no baixo ventre (BV). A relação sexual (32\%) e o esforço físico (29\%) foram citados como fatores de piora da dor. Identificaram-se, entre os fatores de melhora, o uso de analgésicos (47\%) e o repouso (25\%). Durante o período menstrual, $70 \%$ das mulheres relataram sentir dor mais intensa.

Na percepção das participantes, parto (25\%), estado emocional (14\%), estresse (14\%) e trauma por abuso físico e/ou sexual (7\%) foram os fatores que contribuíram para desencadear a DPC. Constatou-se que $28 \%$ realizaram cirurgia para o tratamento da dor. Para 57\% dessas, a vida não mudou com o tratamento cirúrgico. A disúria foi mais frequente nas mulheres com DPC (48\%; $p<0,01)$. Em relação à atividade física, os resultados apontaram que a maioria das mulheres de ambos os 
grupos não praticava exercícios físicos (68\% com DPC e $66 \%$ sem DPC, $p=0,83$ ).

$\mathrm{Na}$ avaliação da qualidade de vida, identificou-se, no grupo com dor, que as dimensões que tiveram escores inferiores a 50 foram saúde mental $(47,9 \pm 17,9)$, vitalidade $(40,9 \pm 21,7)$, dor $(39,3 \pm 15,6)$, aspectos físicos $(33,5 \pm 38,7)$ e aspectos emocionais $(29,3 \pm 37,3)$. O componente da saúde mental apresentou escore inferior $(42,72 \pm 10,2)$ em relação ao componente da saúde física
$(45,62 \pm 10,9)$. Ao comparar os dois grupos, evidenciou-se que mulheres com DPC apresentaram escores inferiores em todas as dimensões avaliadas pelo SF-36 $(p<0,05)$, exceto na dimensão saúde mental $(p=0,0530)$ (Tabela 2). A diferença foi ainda maior $(p \leq 0,003)$ nas dimensões capacidade funcional, aspectos físicos, dor, estado geral de saúde, vitalidade e aspectos emocionais (Tabela 2).

Verificou-se correlação negativa $(p=0,017)$ entre a intensidade da dor e a dimensão dor do SF-36 (Tabela 3).

Tabela 2: Comparação de médias dos escores das dimensões do SF-36 de mulheres com e sem dor pélvica crônica. Goiânia, GO, Brasil, 2009-2010.

\begin{tabular}{|c|c|c|c|}
\hline \multirow{2}{*}{ SF-36 } & Grupo com DPC (n=50) & Grupo sem DPC $(n=50)$ & \multirow{2}{*}{$p^{*}$} \\
\hline & Média ( $\pm D P$ ) & Média ( $\pm D P$ ) & \\
\hline \multicolumn{4}{|l|}{ Dimensões } \\
\hline Capacidade funcional & $56,5( \pm 22,0)$ & $78,1( \pm 20,7)$ & $<0,0001$ \\
\hline Aspectos físicos & $33,5( \pm 38,7)$ & $71,0( \pm 32,3)$ & $<0,0001$ \\
\hline Dor & $39,3( \pm 15,6)$ & $61,1( \pm 21,6)$ & $<0,0001$ \\
\hline Estado geral de saúde & $53,1( \pm 20,9)$ & $66,9( \pm 21,3)$ & 0,0015 \\
\hline Vitalidade & $40,9( \pm 21,7)$ & $54,6( \pm 23,5)$ & 0,0031 \\
\hline Aspectos sociais & $52,8( \pm 24,1)$ & $63,5( \pm 26,3)$ & 0,0358 \\
\hline Aspecto emocional & $29,3( \pm 37,3)$ & $60,0( \pm 39,8)$ & 0,0001 \\
\hline Saúde mental & $47,9( \pm 17,9)$ & $55,3( \pm 19,7)$ & 0,0530 \\
\hline \multicolumn{4}{|l|}{ Componentes } \\
\hline Saúde física & $45,62( \pm 10,9)$ & $69,29( \pm 7,1)$ & 0,0111 \\
\hline Saúde mental & $42,72( \pm 10,2)$ & $58,34( \pm 4,2)$ & 0,0468 \\
\hline
\end{tabular}

DPC: Dor Pélvica Crônica; * teste t de Student

Tabela 3: Correlação entre as dimensões do SF-36 e a intensidade da dor de mulheres com DPC. Goiânia, GO, Brasil, $2009-2010$.

\begin{tabular}{ccc}
\hline Dimensões SF-36 & \multicolumn{2}{c}{ Intensidade da Dor } \\
\cline { 2 - 3 } & $\boldsymbol{r}$ & $\boldsymbol{p}^{*}$ \\
\hline Capacidade funcional & $-0,146$ & 0,310 \\
Aspectos físicos & 0,099 & 0,494 \\
Dor & $-0,337^{*}$ & 0,017 \\
Estado geral de saúde & $-0,156$ & 0,818 \\
Vitalidade & $-0,033$ & 0,280 \\
Aspectos sociais & $-0,070$ & 0,627 \\
Aspecto emocional & 0,082 & 0,571 \\
Saúde mental & $-0,174$ & 0,227 \\
\hline
\end{tabular}

* Correlação de Spearman significante $p<0,05$.

\section{DISCUSSÃO}

A dor pélvica crônica é uma condição que afeta a saúde das mulheres ao longo de sua vida e que necessita ser melhor compreendida no contexto do cuidar dessas pessoas. Os resultados do presente estudo, assim como de outros sobre a temática apontam que em geral as mulheres com DPC estão em uma faixa etária entre 15 e 73 anos $^{(6,14)}$. No grupo investigado, a intensidade da dor foi classificada como moderada e intensa e, assim como descrito em outros estudos, essas mulheres demoraram a perceber a dor como desconforto que necessitava de acompanhamento e tratamento clínico contínuo, para a promoção do seu bem-estar ${ }^{(3,5,22)}$.

Assim como identificado no presente estudo, a maioria das mulheres com DPC faz uso de analgésicos diários e refere que essa intervenção é o principal fator 
de melhora da dor ${ }^{(23)}$. A disúria foi mais frequente entre mulheres com DPC do que no grupo controle. Esse achado está em conformidade com outro estudo, em que os autores referiram associação da DPC com sintomas urinários como disúria e sensação dolorosa ${ }^{(24)}$.

A avaliação da qualidade de vida relacionada à saúde evidenciou entre mulheres com DPC o impacto negativo da dor em sua satisfação com a vida, interferindo no bemestar físico e emocional, reduzindo a produtividade no trabalho e atividades diárias ${ }^{(3,8,15)}$. Apesar de no presente estudo não ter sido observada diferença estatisticamente significativa nos escores da dimensão saúde mental nos dois grupos, verificou-se que, em todas as dimensões avaliadas, os valores das mulheres com DPC foram inferiores. Acredita-se que, nesse caso, o tamanho da amostra pode ter influenciado o resultado.

Esses resultados confirmam as evidências de outros estudos que identificaram que mulheres com DPC, geralmente, apresentam escores inferiores de $\mathrm{QV}^{(8,15-16)}$. Pesquisadores descreveram que o aumento da dor diminui a QV, sendo que os domínios mais afetados foram os aspectos físicos e saúde mental ${ }^{(22)}$. Em outro estudo sobre DPC e $\mathrm{QV}^{(23)}$, os pesquisadores relataram que a dor física ainda é o principal problema entre as mulheres, após longo período de acompanhamento dessa condição crônica. Outro aspecto identificado é o fato de que mulheres com DPC perceberam efeito negativo em sua saúde física e mental, com declínios significativos na vida produtiva e social ${ }^{(3,22)}$.

A dimensão aspecto social avaliou problemas na saúde física ou alterações emocionais que interferiram nas atividades sociais. Nesse estudo, foi observado prejuízo significante na vida social das mulheres com DPC, assim como estudos anteriores ${ }^{(8,15)}$. A dimensão vitalidade analisou a percepção subjetiva quanto ao grau de disposição de saúde, se as pessoas são cheias de vida, com energia ou cansadas e esgotadas. As mulheres com DPC apresentaram vitalidade reduzida. Denota-se que mulheres com DPC, mesmo lidando com a presença da dor, continuam se envolvendo em atividades sociais e habituais da vida, embora possam não ser compreendidas por aqueles com quem convivem ${ }^{(22)}$.

Entre as limitações do estudo está o fato de não ter havido possibilidade de pareamento com o grupo controle, pois durante o processo de coleta de dados, verificaram-se diferenças em termos de faixa etária das mulheres desse grupo. Além disso, é importante ressaltar que o delineamento deste estudo foi transversal, o que permitiu estabelecer associações entre as variáveis e não relações de causa e efeito. No entanto, este estudo sustenta a observação de outros estudos brasileiros de que a DPC afeta a QV de mulheres brasileiras ${ }^{(8,15-16)}$.

\section{CONCLUSÃO}

Os resultados demonstraram que a dor pélvica crônica teve impacto negativo na qualidade de vida com prejuízos para a saúde física e mental das mulheres. Embora os resultados não possam ser generalizados, apontam lacunas do conhecimento sobre esse sintoma crônico, às vezes negligenciado e pouco explorado, porém vivenciado frequentemente pelas mulheres.

Nesse contexto, é necessário ampliar e aprofundar a discussão sobre os aspectos clínicos, terapêuticos e as demandas assistenciais para o cuidado de mulheres com DPC. Para isso, é necessária a adoção de modelos de atenção à saúde que considerem as necessidades das mulheres e utilizem intervenções em saúde de modo compreensivo e holístico para o alívio da dor com ênfase na promoção do bem-estar e preservação da funcionalidade global das mulheres.

Em síntese, a produção de evidências sobre essa temática contribuirá para o aprimoramento do modelo de atenção à saúde de mulheres que vivenciam essa condição crônica, para que tenham o tratamento adequado que minimize o sofrimento, vivenciado muitas vezes, de modo solitário e silencioso. Para aprofundar a compreensão da importância clínica da DPC, sugere-se a realização de estudos epidemiológicos de base populacional para identificar a prevalência de dor pélvica crônica em mulheres brasileiras e identificar os principais 
fatores associados a essa condição crônica. Além disso, é

fundamental compreender a experiência vivenciada por

\section{REFERÊNCIAS}

1. Royal College of Obstetricians and Gynecologists. The initial management of chronic pelvic pain. Green-top Guideline №

41. London: RCOG, 2012. 16p.

2. Willians RE, Hartmann KE, Steege JF. Documenting the current definitions of chronic pelvic pain: implications for research. Obstet Gynecol. 2004;103(4):686-91.

3. Grace V, Zondervan K. Chronic pelvic pain in women in New Zealand: comparative well-being, comorbidity, and impact on work and other activities. Health Care Women Int. 2006;27(7):585-99.

4. Davies L, Gangar KF, Drummond M, Saunders D, Beard RW. The economic burden of intractable gynaecological pain. J Obstet Gynecol. 1992;12(2):46-54.

5. Abercrombie PD, Learman LA. Providing holistic care for women with chronic pelvic pain. J Obstet Gynecol Neonatal Nurs. 2012;41(5):668-79.

6. Howard FM. Chronic pelvic pain. Obstet Gynecol. 2003;101(3):594-611.

7. TerKuile MM, Weijenborg PT, Spinhoven P. Sexual functioning in women with chronic pelvic pain: the role of anxiety and depression. J Sex Med. 2010;7(5):1901-10.

8. Romão $A P$, Gorayeb R, Romão GS, Poli Neto OB, Reis FJ, Silva $J C R$, Nogueira AA. High levels of anxiety and depression have a negative effect on quality of life of women with chronic pelvic pain. Int J Clin Pract. 2009;63(5):707-11.

9. Latthe P, Latthe M, Say L, Gulmezoglu M, Khan KS. WHO Systematic review of prevalence of chronic pelvic pain: a neglected reproductive health morbidity. BMC Public Health. 2006;6:177.

10. Chao MT, Abercrombie PD, Duncan LG. Centering as a model for group visits among women with chronic pelvic pain. J Obstet Gynecol Neonatal Nurs. 2012;41(5):703-10.

11. Alebtekin A. Prevalence of chronic pelvic pain among women: an updated review. Pain Physician. 2014;17(2):E141-7. 12. Silva GPOG, Nascimento LA, Michelazzo D, Junior FFA, Rocha MG, Rosa-e-Silva JC, dos Reis FJC, Nogueira AA, PoliNeto $\mathrm{OB}$. High prevalence of chronic pelvic pain in women in Ribeirão Preto, Brazil and direct association with abdominal surgery. Clinics (Sao Paulo). 2011;66(8):1307-12.

13. Cheong $Y$, Stones WR. Chronic pelvic pain: aetiology and therapy. Best Pract

Clin Obstet Gynaecol. 2006;20(5):695-711.

14. Zondervan KT, Yudkin PL, Vessey MP, Jenkinson CP, Dawes MG, Barlow DH, Kennedy SH. The community prevalence of chronic pelvic pain in women and associated illness behaviour. Br J Gen Pract. 2001;51(468):541-7.

15. Barcelos PR, Conde DM, De Deus JM, Martinez EZ. Qualidade de vida de mulheres com dor pélvica crônica: um estudo de corte transversal analítico. Rev. Bras. Ginecol.

Obstet. 2010;32(5):247-53. essas mulheres em relação ao enfrentamento da dor pélvica crônica em suas vidas.

16. Souza CA, Oliveira LM, Scheffel C, Genro VK, Rosa V, Chaves MF et al. Quality of life associated to chronic pelvic pain is independent of endometriosis diagnosis-across-sectional survey. Health Qual Life Outcomes. 2011;10:9-41.

17. Barbetta PA. Estatística aplicada às ciências sociais. 5 th ed. Florianópolis: Editora da UFSC; 2002.

18. Briggs $M$, Closs JS. A descriptive study of the use of visual analogue scales and verbal rating scales for the assessment of postoperative pain in orthopedic patients. J Pain Symptom Manage. 1999;18(6):438-46.

19. Ware JE, Sherbourne CD. The MOS 36-item short-form health survey (SF-36). I. conceptual framework and item selection. Med Care. 1992;30(6):473-83.

20. Ciconelli RM, Ferraz MB, Santos W, Meinão I, Quaresma MR. Tradução para a línguaportuguesa e validação do questionário genérico de avaliação de qualidade de vida SF-36 (Brasil SF-36). Rev. bras. reumatol. 1999;39(3):143-50. 21. Neelakantan D, Omjole F, Clark TJ, Gupta JK, Khan KS. Quality of life instruments in studies of chronic pelvic pain: a systematic review. J Obstet Gynaecol. 2004;24(8):851-8.

22. Haggerty CL, Schulz R, Ness RB; PID Evaluation and Clinical Health Study Investigators. Lower quality of life among women with chronic pelvic pain after pelvic inflammatory disease. Obstet Gynecol. 2003;102(5 Pt 1):934-9.

23. Louise C, Ayers S, Nala K, Penny J. Chronic pelvic pain and quality of life after laparoscopy. Eur J Obstet Gynecol Reprod Biol. 2007;132(2):214-9.

24. Nogueira AA, Reis FJC, Poli Neto BO. Abordagem da dor pélvica crônica em mulheres. Rev. Bras. Ginecol. Obstet. 2006;28(12):733-40.

Recebido: 12/12/2013.

Aceito: 16/07/2014.

Publicado: 30/09/2015. 